\title{
POTENCIALIZANDO O ENSINO DE MATEMÁTICA ATRAVÉS DE OBJETOS DE APRENDIZAGEM
}

\section{POTENTIALIZING MATH TEACHING THROUGH LEARNING OBJECTS}

\author{
LAVOR, Otávio Paulino ${ }^{1}$ \\ MARTINS, Kaliane Morais de Lucena²
}

\section{RESUMO}

O processo de ensino e aprendizagem atravessa dificuldades e uma das causas é a falta de motivação do público envolvido. Como as tecnologias estão cada vez mais presentes nas escolas brasileiras, abrem-se possibilidades para o ensino da matemática e a demanda por sua incorporação no processo de ensino e aprendizagem na sala de aula se torna comum e tende a fazer parte do cotidiano da escola e dos alunos. Diante disto, propõe-se o uso dos objetos de aprendizagem, que são recursos tecnológicos reutilizáveis, como instrumentos didáticos nas aulas de matemática a fim de superar barreiras e dificuldades de aprendizagem. A pesquisa analisa a importância de trabalhar com objetos de aprendizagem para o ensino de matemática e para isso, questiona os professores de matemática do ensino médio de uma escola paraibana. Na constituição desta pesquisa, realizamos uma análise do processo de reflexão desse professor sobre sua própria prática pedagógica e uma avaliação dos possíveis benefícios trazidos pelo uso de atividades desenvolvidas em ambientes virtuais e o uso de recursos tecnológicos no processo de ensino aprendizagem de matemática como os objetos de aprendizagem. Constatou-se que os professores tiveram formação sem o uso de tecnologias, mas estão lecionando para alunos imersos em ambiente tecnológico e que os mesmos não conheciam nem trabalhavam com os objetos de aprendizagem virtual. A partir daí, uma oficina de objetos de aprendizagem conscientiza os professores com a apresentação dos recursos e como utilizá-los, levando a uma potencialização na construção do conhecimento e no entusiasmo para ensinar e aprender matemática de forma mais significativa e dinâmica.

PaLAVRAS-CHAVE: TIC; Impasses; Motivação; Recursos didáticos; Aprendizagem.

\section{ABSTRACT}

The teaching and learning process go through difficulties and one of the causes is the lack of motivation of the involved public. As the technologies are increasingly present in Brazilian schools, it open possibilities for mathematics teaching and the demand for their incorporation into the process of teaching and learning in the classroom becomes common and tends to be part of the daily life of the school and its students. Given this, it is proposed to use learning

1 Universidade Federal Rural do Semi-árido (UFERSA). Pau dos Ferros, RN, Brasil. ORCID: https://orcid.org/0000-0001-5237-3392 e-mail: otavio.lavor@ufersa.edu.br

2 Universidade Federal do Rio Grande do Norte (UFRN). Pau dos Ferros, RN, Brasil. ORCID: https://orcid.org/0000-0002-1253-4822 e-mail: kaliane@hotmail.com 
DOI: $10.12957 / \mathrm{e}-\mathrm{mosaicos} .2020 .46565$

objects, which are reusable technological resources, as didactic tools in math classes in order to overcome barriers and learning difficulties. The research analyzes the importance of working with learning objects for the teaching of mathematics and for this, questions the high school math teachers of a paraibana school. It was found that teachers have been trained without the use of technologies, but are teaching to students immersed in technological environment. From there, a learning object workshop makes teachers aware of the presentation of resources and how to use them, leading to a potential for building knowledge and enthusiasm for teach and learn.

KeYWORDS: TIC; Impasses; Motivation; Didactic resources; Learning.

\section{INTRODUÇÃO}

As tecnologias digitais estão a cada dia se tornando um mediador cultural cada vez mais presente nas escolas brasileiras e um recurso muito importante no processo de ensino-aprendizagem, ocupando um papel de destaque no auxílio ao aluno na construção do conhecimento. $O$ uso dessas tecnologias nas últimas décadas tem proporcionado muitas pesquisas e estudos no processo de ensino e aprendizagem. Dentro do contexto da revolução tecnológica ocorrida nos últimos vinte anos, no ensino está adentrando o ambiente escolar com fins educativos, livrarias e bancas de revistas costumam dedicar prateleiras à divulgação de produtos multimídias. As tecnologias da Informação e Comunicação (TICs) deram um novo suporte ao ato de ensinar e aprender. E as TICs estão cada vez mais frequentes no cotidiano dos alunos, professores, escola e principalmente no ensino da matemática.

Hoje não se questiona mais sobre a utilização das tecnologias na escola, como podem ser usados os recursos tecnológicos no processo ensino aprendizagem, todavia, sobre quais as tecnologias são mais apropriadas e como as mesmas podem ser utilizadas, pois uma grande parte dos recursos tecnológicos das escolas ainda tem se restringido a usos pouco interativos com programas em que o aluno somente acompanha um conteúdo e em seguida realiza testes e exercícios. Outros usos estão relacionados a aspectos técnicos como planilhas eletrônicas ou pesquisas na Internet.

Os professores têm acessado a Internet e outros recursos tecnológicos para enriquecer e apoiar nas suas aulas, mas não utilizam 0 real potencial que as tecnologias podem oferecer na construção do conhecimento. Alguns autores, como Carraher (1992), defendem o computador como uma ferramenta pedagógica desde que seu uso oportunize descobertas e desafios com as atividades que instiguem os alunos a pensarem, dando possibilidades tanto para erros como acertos, e não meramente explique, corrijam ou reforce respostas. Portanto o uso eficaz desse mediador simbólico no processo de ensino requer tanto o conhecimento da "Psicologia da Aprendizagem e do Desenvolvimento, bem como as pesquisas sobre os conceitos específicos na área do conhecimento em consideração" (CARRAHER, 1992, p. 199). Outros autores como Oliveira (1998), Valente (1993), Santos et al. (2005) e 
DOI: $10.12957 /$ e-mosaicos.2020.46565

Nascimento (2007) têm também destacado estudos sobre a eficácia do uso das ferramentas digitais na educação, ampliação e discussão sobre a oferta de conteúdos digitais para os professores utilizarem no processo ensino aprendizagem.

Entretanto, com o avanço nas tecnologias da informação e da comunicação continuamos a presenciar nas escolas que aprender e ensinar matemática não são tarefas fáceis e muitos são os medos, as queixas, as dificuldades para enfrentar o desafio de ensinar bem e aprender os conceitos matemáticos. Assim muitos são os processos de ensino e de aprendizagem, mas é preciso considerar três variáveis fundamentais e as necessárias relações que se estabelecem entre elas: aluno, professor e conhecimento matemático. Neste texto apresentaremos não só a relação de professor e aluno, mas uma interface a mais, os objetos de aprendizagem como mediadores de um saber de forma lúdica, crítica e criativa. Devemos pensar no conhecimento matemático como ciência viva, aberta à incorporação de novos conhecimentos de forma que o aluno possa aprender apreendendo e contextualizando os conhecimentos apreendidos.

Nessa perspectiva muitos são os obstáculos envolvidos na construção dos conceitos matemáticos para que se possa compreender como acontece sua aprendizagem pelos alunos. Os obstáculos não estão presentes somente na dificuldade, complexidade de aprender os conteúdos, mas são determinados também pelas características cognitivas, sociais e culturais de quem aprende e pelas formas como são ensinados. A matemática está na vida de todos nós, no entanto é ensinada como algo abstrato que não existe que ninguém vivencia no seu cotidiano.

Todavia é preciso novas formas e novas interfaces para mediar esse ensinar e aprender. Nesse sentido as TICs podem contribuir garantindo simulações que possam observar regularidades, generalizar e transferir tais conhecimentos a outros contextos, pois um conhecimento só torna-se pleno quando pode ser aplicado em situações diferentes das que lhe deram origem, podendo assim estabelecer conexões e possa compreender os conteúdos matemáticos e aplicá-los na resolução de problemas.

Este trabalho discute alternativas para essas dificuldades, procurando investigar a validade do uso dos Objetos de Aprendizagem (OA) como ferramenta pedagógica mediadora dentro de um conteúdo matemático, procurando estreitar as relações e comunicações nas aulas de matemática já que as mesmas são quase inexistentes, pois o processo de ensino é feito de forma bem tradicional. Analisar e refletir sobre essa interação são importantes para repensar o ensino e aprendizagem de forma inovadora onde o professor e aluno tornam-se sujeitos participantes do processo, o professor como mediador e o aluno sendo sujeito atuante na construção do seu conhecimento, para realizar esse trabalho foi feito um estudo bibliográfico combinado com um estudo empírico. O trabalho apresenta a definição de Objetos de Aprendizagem e sua aplicação no ensino de matemática, o uso das TICs como ferramentas suporte para a aprendizagem, com a ideia de que os objetos de aprendizagem, que são software que podem ser trabalhados em computadores, tablete, smartfone, são elementos 
DOI: $10.12957 /$ e-mosaicos.2020.46565

mediadores do processo de aprendizagem. Nessa perspectiva, recorremos à teoria vigotskiana que define aprendizagem como um processo pelo qual os indivíduos adquirem habilidades, valores e informações em contato com a realidade e outras pessoas (VYGOTSKY, 1991).

\section{FUNDAMENTAÇÃO TEÓRICA}

As inovações tecnológicas têm causado profundas mudanças na sociedade como uma força transformadora presente em quase todos os âmbitos de atividades social, inclusive o da Educação. A escola está em um contexto onde as novas tecnologias se fazem cada vez mais presente e necessitam ser utilizadas, pois o modo de aprender de hoje é diferente do que foi no passado. Vygotsky (1998) expõe que o impacto do uso de instrumentos sobre os homens é importante não porque os ajudam a se relacionar mais ativamente com o seu ambiente como também aos importantes efeitos que o uso de instrumentos tem sobre as relações internas e funcionais no interior do cérebro humano.

Na sala de aula o professor já não consegue mais, de maneira tradicional, despertar o interesse, a motivação de seus alunos. Então, faz-se necessário haver questionamentos, reflexões e mudanças sobre atividades educativas que podem tornase interessantes aos alunos como o uso de novos instrumentos, Objetos de aprendizagem, fazendo com que o aluno pense, questione e desenvolva a sua autonomia. Berg escreve a respeito:

assim como os instrumentos de trabalho mudam historicamente, os instrumentos do pensamento também se transformam historicamente. E assim como novos instrumentos de trabalho dão origem a novas estruturas sociais, novos instrumentos de pensamento dão origem a novas estruturas mentais (...). Para Vygotsky, todavia, tanto as estruturas sociais como as estruturas mentais têm de fato raízes históricas muito definidas, sendo produtos bem específicos de níveis determinados do desenvolvimento dos instrumentos (BERG in: Vygotsky, 1988, p. 177).

Sendo assim o computador, a Internet, os objetos de aprendizagem, a TV Multimídia entre outros recursos digitais, pode ser considerada atualmente os novos instrumentos de trabalho que modificam as estruturas sociais e através dos quais introduz novas formas de pensar modificando as estruturas mentais e transformando a relação entre o ser humano e o meio ao qual está inserido. É necessário que os professores reflitam sobre o uso das novas tecnologias, mude o seu perfil, redefina 0 seu papel, amplie seus conhecimentos para poder lidar com as transformações desse novo contexto. Para Moran (2013, p. 1) "a escola pode ser um espaço de inovação, de experimentação saudável de novos recursos e que não precisamos romper com o passado, mas programar mudanças e observá-las com paridade e maturidade". Dessa 
DOI: $10.12957 /$ e-mosaicos.2020.46565

forma, compreende-se que mudanças devem surgir para que a escola seja um espaço de diálogo favorecedor da aprendizagem.

\section{TeCNOLOGIAS de INFORMAÇÃo e CoMUniCAÇÃo (TICS)}

As Tecnologias da Informação e Comunicação (TICs) deram um novo suporte ao ato de ensinar e aprender. Elas estão cada vez mais presentes no cotidiano de alunos e professores. Diante do cenário atual escolar brasileiro, os educadores necessitam cada vez mais de alternativas pedagógicas que auxiliem o processo de ensino e aprendizagem. A informática e o computador podem ser recursos altamente eficientes para a melhoria desse processo.

Estamos, atualmente, vivendo uma evolução no ensino, que tem como aliado as Tecnologias de Informação e Comunicação, trazendo consigo suas inúmeras vantagens e possibilidades de uso voltadas para o processo de ensino aprendizagem, compartilhado desta ideia Silva et. al. (2007) afirmam que:

As sociedades contemporâneas têm grandes desafios a enfrentar pelo fato do conhecimento ter se tornado o centro dos processos de transformação social, consequentemente, a educação assume, neste contexto, um importante papel para além da reprodução e promoção social. Aliada as tecnologias à educação tenta enfrentar estes desafios quando utiliza alternativas importantes para o processo de reflexão e (re)leituras das diferentes formas de conhecimento que são disseminados pelas novas tecnologias da informação (TIC) como são chamadas (SILVA et.al., 2007, p. 8).

As TICs possibilitaram um novo suporte educacional, recheadas de possibilidades, nas quais os educadores podem encontrar e trocar alternativas pedagógicas que os auxiliem no ato de ensinar e aprender, efetivando um processo de compartilhamento no qual todos saem ganhando. Assim, a colaboração pode facilitar o processo de ensino, e transformá-lo, tornando-o mais criativo, inovador e motivador.

O uso do computador na educação objetiva a integração deste no processo de aprendizagem dos conceitos curriculares em todas as modalidades e níveis de ensino, podendo desempenhar papel de facilitador entre o aluno e a construção do seu conhecimento. Ele enfatiza a necessidade de os docentes estarem preparados para realizar atividades computadorizadas com seus alunos (VALENTE, 1999).

O setor educativo precisa explorar e construir conhecimentos segundo as necessidades de seu desenvolvimento numa articulação em que a unidade escolar assume o papel de mobilizadora de transformações e o professor, o papel de promotor da aprendizagem. 
DOI: $10.12957 /$ e-mosaicos.2020.46565

O professor é o principal ator de qualquer processo de escolha ou introdução de inovações tecnológicas na escola. Para que haja mudanças na qualidade do seu trabalho docente, é necessário que possa refletir e entender as suas escolhas pedagógicas, a importância que dá aos diferentes componentes curriculares para, então, analisar de que modo as diversas tecnológicas poderão auxiliá-lo no processo de ensino-aprendizagem (VALENTE, 1999).

No Ensino da Matemática, torna-se evidente que ao usar o computador, seja com uma simples apresentação de slides ou algo mais complexo como softwares matemáticos, os alunos retêm forma mais concreta o conhecimento.

Os Parâmetros Curriculares Nacionais de Matemática (BRASIL, 1998, p. 44) trazem em seu texto indicações de como e para quais finalidades o computador pode ser usado nas aulas de Matemática:

- como fonte de informação, poderoso recurso para alimentar o processo de ensino e aprendizagem;

- Como auxiliar no processo de construção do conhecimento;

- Como meio para desenvolver autonomia pelo uso de softwares que possibilitem pensar, refletir e criar soluções;

- Como ferramenta para determinadas atividades uso de planilhas eletrônicas, processadores de texto, banco de dados, etc.

A finalidade de seu uso está associada ao objetivo a ser alcançado com o conteúdo. Os PCN (1998), em relação aos recursos de softwares, por exemplo, argumentam que seu sucesso depende, em grande parte, da escolha feita pelo professor, devendo - o software - ser apropriado ao que se pretende alcançar. Um exemplo dado é o estudo das funções, para o qual há softwares que auxiliam na análise do comportamento gráfico, levando 0 aluno a fazer conjecturas sobre 0 comportamento da função, suas possibilidades de variação, etc.

Ainda, segundo os PCN (1998), uma das vantagens do uso do computador para a aula é que, além de possibilitar a criação de ambientes de aprendizagem nos quais os alunos têm condições de experimentar e conferir suas hipóteses permite a interação entre os alunos. No entanto, nos PCN, não se estende a discussão acerca da interação e dos benefícios que ela poderá trazer para a aprendizagem do aluno e para a própria produção do conhecimento.

Nessa perspectiva utilizamos Objetos de Aprendizagem (OA), como recurso didático nas aulas de matemática demonstrando sua importância para o processo ensino aprendizagem e podendo investigar o seu uso junto aos professores no processo de ensino e aprendizagem de matemática potencializando a construção do conhecimento de uma forma dinâmica e viva. 
DOI: $10.12957 / \mathrm{e}-\mathrm{mosaicos} .2020 .46565$

\section{Objetos de APRendizagem}

Os Objetos de Aprendizagem podem ser vistos como componentes ou unidades digitais, catalogados e disponibilizados em repositórios na Internet para serem reutilizados para o ensino.

Conforme o Comitê de Padronização das Tecnologias de Aprendizagem (Learning Technology Standards Committee - LTSC), um Objeto de Aprendizagem é definido como "qualquer entidade, digital ou não digital, que pode ser usado para aprendizado, educação ou treinamento". (IEEE, 2002, p. 3). Dessa forma, se compreende que os objetos de aprendizagem são recursos que facilitam o processo de ensino e aprendizagem criando estímulo e reflexão ao estudante.

Segundo Singh citado por Bettio e Martins (2002), um objeto de aprendizagem para ser bem estruturado é dividido em três partes bem definidas: 1) Objetivos: Esta parte demonstra ao aprendiz o que ele poderá aprender a partir do estudo desse objeto, 2) Conteúdo instrucional: material didático necessário para que no término o aluno possa atingir os objetivos. 3) Prática e FeedBack: em cada final de utilização, o aprendiz verifica se o seu desempenho atingiu as expectativas. As características dos objetos de aprendizagem possuem duas perspectivas: a pedagógica e a técnica. As características relacionadas à dimensão pedagógica se referem aos OA que facilitem o trabalho dos envolvidos, visando à aquisição do conhecimento (DIAS et al., 2009). Dessa forma, são considerados aspectos pedagógicos importantes (GALAFASSI et al., 2013, p. 43):

Interatividade: indica se há suporte às consolidações e ações mentais, requerendo que o aluno interaja com o conteúdo do OA de alguma forma, podendo ver, escutar ou responder algo.

Autonomia: indica se os objetos de aprendizagem apoiam a iniciativa e tomada de decisão.

Cooperação: indica se há suporte para os alunos trocarem opiniões e trabalhar coletivamente sobre o conceito apresentado.

Cognição: refere-se às sobrecargas cognitivas alocadas na memória do aluno durante o processo de ensino-aprendizagem.

Afetividade: refere-se aos sentimentos e motivações do aluno com sua aprendizagem e durante a interação com o OA.

As características técnicas se referem às questões tecnológicas. A seguir, serão apresentadas as características técnicas dos OAs (BRAGA; MENEZES, 2015, p. 27-28):

Disponibilidade: indica se o objeto está disponível para ser utilizado.

Acessibilidade: indica se o objeto pode ser acessado por diferentes tipos de usuários (ex: idosos, deficientes visuais etc), em diferentes lugares (ex: lugares com acesso à internet, lugares sem acesso à internet etc) e por diferentes tipos de dispositivos (ex: computadores, celulares, tablets etc) 
DOI: $10.12957 /$ e-mosaicos.2020.46565

Confiabilidade: indica que o OA não apresenta defeitos técnicos ou problemas no conteúdo pedagógico.

Portabilidade: indica se o OA pode ser transferido (ou instalado) para diferentes ambientes, como, por exemplo, diferentes tipos de AVAs ou sistemas operacionais.

Facilidade de instalação: indica se o OA pode ser facilmente instalado caso ele exija esse recurso.

Interoperabilidade: medida de esforço necessário para que os dados dos OAs possam ser integrados a vários sistemas.

Usabilidade: indica a facilidade de utilização dos Oas por alunos e professores.

Manutenibilidade: é a medida de esforço necessária para alterações do OA.

Granularidade: De maneira geral, a palavra granularidade origina-se da palavra grão, sendo que quanto maior o número de grãos de um sistema maior a sua granularidade. Trazendo este conceito para o âmbito dos objetos de aprendizagem, a granularidade é a extensão à qual um OA é composto por componentes menores e reutilizáveis.

Agregação: indica se os componentes do OA (grãos) podem ser agrupados em conjuntos maiores de conteúdos como, por exemplo, as estruturas tradicionais de um curso.

Durabilidade: indica se o OA se mantém intacto quando o repositório em que ele está armazenado muda ou sofre problemas técnicos.

Reusabilidade: indica as possibilidades de reutilizar os OAs em diferentes contextos ou aplicações.

A reusabilidade é a principal característica do OA e pode ser influenciada por todas as demais. Vale ter em conta que nem todo objeto de aprendizagem apresenta todas as características listadas. No entanto, quanto mais características ele tiver, maior a sua capacidade de reutilização. Essas características devem ser utilizadas para avaliar o nível de reuso de um OA.

Os objetos com essas características são armazenados em grandes bases de dados disponíveis na Internet, denominados de repositórios, que permitem o armazenamento, o desenvolvimento e o relacionamento existente entre os objetos de aprendizagem, podendo ser vistos como grandes bibliotecas digitais que facilitam a pesquisa, a análise e a seleção de objetos de aprendizagem para uso na educação. Alguns dos Repositórios de Objetos de Aprendizagem encontrados são Portal Educacional do Estado do Paraná; Dia-a-dia Educação; CESTA (Coletânea de Entidades de Suporte ao uso de Tecnologia na Aprendizagem); RIVED (Rede Internacional Virtual de Educação), PROATIVA (Grupo de Pesquisa e Produção de Ambientes Interativos e Objetos de Aprendizagem), Portal do Professor do MEC entre outros.

De acordo com Braga e Menezes (2015, p.32):

Os Os OAs podem ser vistos como unidades digitais catalogadas, disponibilizados em repositórios na Internet, para serem reutilizados. Os 
DOI: $10.12957 /$ e-mosaicos.2020.46565

repositórios, por sua vez, podem ser vistos como bancos de OAs, em que estes estão organizados e catalogados de maneira a facilitar sua busca e utilização.

Assim, um objeto de aprendizagem pode possibilitar ao estudante que ele construa o seu próprio aprendizado, usando o seu pensamento gradualmente, construindo significados referentes ao que está estudando, e para isso, deve oferecer contribuições, no sentido de aproveitar do educando os saberes prévios, facilitando a aprendizagem. Um dos trabalhos com OA que teve resultado positivo que vem ao encontro da aprendizagem dos alunos, foi "A análise da dialética ferramenta-objeto no objeto de aprendizagem "potencializando o seu conhecimento", Bonoto (2009) referência que se o OA estiver devidamente de acordo com a intenção do planejamento do professor, é um recurso que oportuniza a "criação de espaços de análise, formulação de hipóteses e generalização por meio da exploração de situações personalizadas".

\section{Metodologia}

A metodologia fundamentou-se em uma abordagem qualitativa de caráter descritivo que nas palavras de Gamboa (1991, p. 91-115), "é uma característica do paradigma crítico-dialético de caráter descritivo porque está voltado para a busca de qualificação das práticas pedagógicas e curriculares da instituição escolar".

A pesquisa, adotando a abordagem qualitativa, buscou realizar um estudo de caso fazendo uso de técnicas combinadas de coleta de dados com o propósito de obter descrições que representem a compreensão e interpretação de fatos relatados em entrevistas semiestruturadas e em fenômenos observados pelo pesquisador. Esta definição se baseia no fato de ser o estudo de caso uma das maneiras de se realizar pesquisa qualitativa com o intuito de se ter um maior aprofundamento na compreensão do comportamento de uma unidade social com relação a algo a ser estudado, entendendo uma determinada situação vivenciada e descrevendo-a, ou seja, penetrando em uma realidade social (GOLDEMBERG, 2005).

A pesquisa foi realizada junto a professores de Matemática do Ensino Médio (EM) de uma escola pública estadual da cidade de Patos/PB com o objetivo de buscar informações pessoais e profissionais do professor, inclusive no uso do computador, outros recursos digitais e seus conhecimentos em relação aos Objetos de Aprendizagem no processo de ensino aprendizagem de matemática potencializando a construção do conhecimento. O questionário foi trabalhado com sete professores de matemática da escola, a partir de dez questões objetivas e subjetivas, onde procurouse saber sobre o tempo em que leciona com a disciplina de matemática, se durante esse tempo utilizou recursos para aprimorar suas aulas objetivando melhorar 0 processo ensino aprendizagem e qual foi esses recursos, se sabem o que é um objeto de aprendizagem, que ferramentas tecnológicas ou recursos tecnológico já utilizou ou utiliza para trabalhar alguns conteúdo de matemática e para melhorar o processo de 
DOI: $10.12957 / \mathrm{e}-\mathrm{mosaicos} .2020 .46565$

ensino aprendizagem dos alunos, se eles acham que com a utilização desses recursos tecnológicos os alunos se motivavam mais, mostrando interesse e progresso.

Realizou-se uma observação nas respostas em que o professor respondeu sobre OA, com o objetivo de colher impressões sobre a sua interação com o recurso e com o ambiente computacional e suas interfaces. Em seguida, na entrevista, foram colhidas opiniões do professor sobre o papel da tecnologia, em especial o computador, na educação; opiniões suas sobre a possibilidade de uso e da inserção curricular do OA com seus alunos; bem como limitações, dificuldades, no seu entender, sobre o uso desses recursos para o ensino de matemática.

\section{RESULTADOS}

Iniciamos com os dados coletados na pesquisa para fazer uma análise sobre o uso dos recursos digitais no ensino de matemática associado à ferramenta objeto de aprendizagem potencializando a construção do conhecimento. Dos professores que participaram da pesquisa e responderam o questionário, mais de $80 \%$ dos professores está numa faixa etária de mais de 40 anos de idade e com mais ou menos de 20 anos de experiência como professores de matemática atuando em sala de aula. Tratam-se, portanto, de indivíduos que, em sua maioria, tiveram sua formação profissional na era midiática (SANTAELLA, 2004), mas que lidam com indivíduos nascidos na era digital.

Todos que responderam o questionário têm um computador para uso pessoal, sendo que utilizam o seu computador pessoal diariamente. Todos acessam a internet diariamente através do seu computador pessoal ou de outra ferramenta como celular, tablet e etc. Dos entrevistados, mais da metade já fizeram algum curso de formação de informática para a educação ou outro curso de informática básica.

Foi perguntado ao professor se durante o tempo que leciona já utilizou algum recurso para aprimorar suas aulas e desenvolver melhor uma relação entre o processo ensino aprendizagem, todos responderam que já utilizou e ao pedir para citar os recursos utilizados $P_{1}$ e $P_{2}$ responderam que já utilizou jogos, $P_{3}, P_{4}$, e $P_{5}$ respondeu que já utilizou jogos confeccionados pelos próprios alunos, dobraduras, maratonas, enigmas entre outros recursos, os $\mathrm{P}_{6}$ e $\mathrm{P}_{7}$ elencaram quase os mesmos recursos mais acrescentaram que trabalharam com resolução de problemas e vídeos aulas. Observase que os recursos tecnológicos quase não são utilizados para motivar e incentivar o processo de ensino aprendizagem de matemática.

Ao perguntar ao professor se já utilizou alguma ferramenta tecnológica ou recursos tecnológicos para favorecer a melhoria da aprendizagem no ensino de matemática, junto ao seu aluno, dos sete professores menos da metade, apenas 2 professores, afirmaram que usam o computador nas aulas de matemática, mas não especificaram como. Vale ressaltar que praticamente, mais da metade dos professores, 4 dos entrevistados não se sentem seguros no uso do computador na disciplina de Matemática. Justificaram dizendo que o laboratório de informática da escola não fica 
DOI: $10.12957 / \mathrm{e}-\mathrm{mosaicos} .2020 .46565$

disponível quando necessário, a falta de equipamentos foi indicada como um obstáculo para o uso desse recurso por alguns e por outros o fato dos professores não se sentirem seguros no uso desse recurso, apesar de a maioria deles terem dito que já participaram de formações continuada de informática ou fez um curso básico de computação.

Ao perguntar sobre a importância de trabalhar com recursos digitais nas aulas de matemática de forma unânime os professores, todos acham de suma importância trabalhar com recursos digitais nas aulas de matemática, porém a grande maioria não utiliza esses recursos como deveria.

E ao serem questionados sobre o que é um objeto de aprendizagem de matemática, todos apontaram para todo recurso que pode ser aplicado como ferramenta no processo de ensino aprendizagem, e apontaram alguns, mas não eram com a utilização da tecnologia.

Apesar de não conhecer o recurso tecnológico objetos de aprendizagem de matemática e ainda não terem trabalhado com os mesmos em sua sala de aula ou no laboratório de informática da escola, acreditam que eles podem potencializar o processo de ensino aprendizagem e consequentemente o conhecimento dos alunos, por se tratar de recursos que estão muito próximo do que os alunos gostam de fazer e também por ser uma forma de dinamizar as aulas de matemática e os alunos possam enxergar os recursos tecnológicos como uma ferramenta para o estudo e pesquisa.

Os professores também foram indagados se eles tinham interesse ou gostariam de utilizar ferramentas tecnológicas ou recursos tecnológicos nas aulas de matemática para favorecer a aprendizagem de forma significativa, dinâmica e interativa, Todos disseram que sim e dois professores acrescentaram que trabalhar matemática de forma dinâmica e interativa ia favorecer muito para que os alunos se motivassem e aprendesse de forma significativa, pois estaria muito próximo do que eles hoje dominam que é a tecnologias.

Como os professores sinalizaram que não tinham conhecimento de como trabalhar com os objetos de aprendizagem da matemática, foi feita uma apresentação aos mesmos na reunião departamental mostrando a definição e onde encontrar, bem como suas características e como utilizá-los. Também foi feita uma demonstração de um objeto de aprendizagem que trabalhou o conteúdo escolhido por eles, grandezas e medidas. Todos ficaram muito entusiasmados em aplicar o recurso na sala de aula e demonstraram muito interesse pelo recurso para potencializar sua prática e aproximar mais o aluno da matemática e diminuir as dificuldades de aprendizagem.

Diante da experiência didática e das respostas dos professores, pode-se ver que o ensino atravessa impasses por falta de motivação do público envolvido. Tais impasses geram desconforto para experimentar 0 novo e desfavorecem a aprendizagem, o que leva discentes concluírem o ensino médio sem a qualificação desejada para os desafios de provas ou mesmo o exercício de cidadania. 
DOI: $10.12957 /$ e-mosaicos.2020.46565

Esta pesquisa constatou que a formação docente se configura como desatualizada com o meio tecnológico em que o público discente se encontra. Então, a oficina de grandezas e medidas através de objetos de aprendizagem potencializa a formação, bem como abre espaços para multiplicação de saberes e motivação a fim de dar passos na construção de uma aprendizagem significativa em matemática.

\section{CONCLUSÃO}

A participação dos professores de Matemática da Escola, como fonte de pesquisa, apoio e incentivo foi de extrema importância para o desenvolvimento desse trabalho. Analisando as respostas dadas no questionário notamos que os professores estavam receosos sobre o uso dos recursos tecnológicos potencializando o processo de ensino aprendizagem na construção do conhecimento, mas ao mesmo tempo com grandes expectativas de inovar e enriquecer as suas aulas sendo necessário realizar encontros para pesquisas e capacitação nessa área.

Através da pesquisa, análise e seleção de Objetos de Aprendizagem puderam perceber a diversidade e o aumento de recursos que os Repositórios oferecem e com isso iniciamos o Uso da TV Multimídia e do Laboratório Digital nas aulas de matemática. Além de despertar o interesse de Professores de outras áreas com relação ao Uso de Objetos de Aprendizagem em suas aulas e seu interesse em planejar sua prática com uso desse recurso fazendo uso do laboratório digital da escola nas suas aulas, aperfeiçoando a sua prática pedagógica e com isso buscando novas formas de ensinar e também de aprender.

Portanto podemos afirmar que é possível a utilização das Novas Mídias Tecnológicas na Educação para obter conhecimento, desde que bem planejada, tendo objetivos claros e definidos, boas leituras, pesquisas, procurando sempre articular os conteúdos com a mídia a serem trabalhada. Assim os Objetos de Aprendizagem no ensino de matemática potencializando a construção do conhecimento, vai inova e enriquece as aulas de Matemática, auxiliam o processo de ensino e aprendizagem, promovem uma melhora no Ensino e favorecendo a Integração entre os Conteúdos de matemática e aos Recursos Tecnológicos.

\section{REFERÊNCIAS}

BETTIO, R. W., MARTINS, A. Objetos de Aprendizado: Um novo modelo direcionado ao Ensino a Distância. In: IX Congresso Internacional de Educação a Distância, São Paulo, 2002.

BONOTO, D.L.; SOARES, M.A.S.; MARTINS, M.C.M. A Análise da Dialética Ferramenta Objeto no Objeto de Aprendizagem "Potencializando O Seu Conhecimento". In: X Encontro Gaúcho de Educação Matemática, Ijuí, 2009. 
DOI: $10.12957 /$ e-mosaicos.2020.46565

BRAGA, J., MENEZES L. Introdução aos objetos de aprendizagem. Em: BRAGA, J. (Org.) Objetos de Aprendizagem Volume 1: introdução e fundamentos. Santo André, UFABC: 2015

BRASIL. Secretaria de Educação Fundamental. Parâmetros curriculares nacionais: Matemática. Secretaria de Educação Fundamental. Brasília: MEC /SEF, 1998.

CARRAHER, D.W. (1992). A aprendizagem de conceitos matemáticos com auxílio do computador. Em: Alencar, E.S. (org.). Novas contribuições da Psicologia aos processos de ensino e aprendizagem. São Paulo: Cortez.

CESTA. Coletânea de Entidades de Suporte ao uso de Tecnologia na Aprendizagem. http://www.cinted.ufrgs.br/CESTA/cestadescr.html . Acesso em 25/01/2019.

DIAS, C. L. et al. Padrões abertos: aplicabilidade em Objetos de Aprendizagem (OAs). In: Simpósio Brasileiro de Informática na Educação (SBIE), Florianópolis, 2009.

GALAFASSI, F.P.; GLUZ, J.C.; GALAFASSI, C. Análise Crítica das Pesquisas Recentes sobre as Tecnologias de Objetos de Aprendizagem e Ambientes Virtuais de Aprendizagem. Revista Brasileira de Informática na Educação, v.21, n.3, p.41-52, 2013.

GAMBOA, S.A.S. A dialética na pesquisa em educação: elementos de contexto. In: FAZENDA, I. (org.). Metodologia da pesquisa educacional. São Paulo, Cortez: 1991.

GOLDEMBERG, Miriam. A Arte de Pesquisar: Como fazer pesquisa qualitativa em Ciências sociais. Rio de Janeiro: Record, 2005.

IEEE Standard for Learning Object Metadata. (2002) In IEEE Std 1484.12.1-2002, Disponível em: https://ieeexplore.ieee.org/stamp/stamp.jsp?tp=\&arnumber=1032843. Acesso em 23/05/2020.

MORAN, José. Educação e Tecnologias: Mudar para valer! Disponível na URL: http://www.netescola.pr.gov.br/netescola/escola/279042018/Gestao.doc Acesso em 05/02/2019.

NASCIMENTO, A. C. A. (2007). Objetos de aprendizagem: entre a promessa e a realidade. Em: Prata, C.L.; Nascimento, A.C.A. (Org.). Objetos de Aprendizagem: Uma proposta de Recurso Pedagógico. Vol. 1 (pp. 135-145). Brasília: Ministério da Educação

OLIVEIRA, R. Informática educativa: dos planos e discursos à sala de aula. Campinas SP: Papirus, 1998.

PARANÁ. Secretaria de Estado da Educação. Superintendência da Educação: diretrizes curriculares de Matemática para as séries finais e para o Ensino Médio. Curitiba. SEED, 2008.

PORTAL DIA-A-DIA EDUCAÇÃO. Disponível em:

http://www.seed.pr.gov.br/diaadia/educadores/. Acesso em 10/20/2019. 
DOI: $10.12957 / \mathrm{e}-\mathrm{mosaicos} .2020 .46565$

PORTAL DO PROFESSOR - MEC Disponível em: http://portaldoprofessor.mec.gov.br/ . Acesso em 05/02/2019.

PROATIVA- Grupo de Pesquisa e Produção de Ambientes Interativos e Objetos de Aprendizagem. Disponível em http://www.proativa.vdl.ufc.br/. Acesso em 10/02/2019.

RIVED - Rede Interativa Virtual de Educação. 2006. Disponível em: http://rived.proinfo.mec.gov.br/site objeto lis.php. Acesso em: 20/01/2019.

SANTAELLA, Lucia. Navegar no ciberespaço: o perfil cognitivo do leitor interativo. São Paulo: Paulus, 2004.

SANTOS, C.; Borges, A.; Ferrari, A. e Ramos, F. (2005). Programação assistida por computador: a experiência da Universidade de Aveiro. Aveiro. Disponível em: http://phoenix.sce.fct.unl.pt/simposio/simposio.htm. Acesso 12/03/2019.

SILVA, E. M.; MOITA, F. M. G. S. C.; SOUSA, R. P. Jogos Eletrônicos: construindo novas trilhas. Campina Grande: EDUEP, 2007.

VALENTE, J.A. A formação de profissionais na área de informática em educação. Em: VALENTE, J.A (Org.). Computadores e conhecimento: repensando a educação. Campinas, SP: Gráfica Central da Unicamp, 1993.

VALENTE, J. A. O computador na sociedade do conhecimento. Campinas, Unicamp/NIED: 1999.

VYGOTSKY, L. S. A Formação Social da Mente: o desenvolvimento dos processos psicológicos superiores; organizadores. Michael Cole (et al), 6a. ed. São Paulo: Martins Fontes, 1998.

VYGOTSKY, L.S. Pensamento e Linguagem. 4ªEd. São Paulo: Martins Fontes, 1991.

Recebido em 10 de novembro de 2019

Aceito em 05 de fevereiro de 2020

A e-Mosaicos Revista Multidisciplinar de Ensino, Pesquisa, Extensão e Cultura do Instituto de Aplicação Fernando Rodrigues da Silveira (CAp-UERJ) está licenciada com uma Licença Creative Commons - Atribuição-NãoComercial 4.0 Internacional.

Os direitos autorais de todos os trabalhos publicados na revista pertencem ao(s) seu(s) autor(es) e coautor(es), com o direito de primeira publicação cedido à e-Mosaicos.

Os artigos publicados são de acesso público, de uso gratuito, com atribuição de autoria obrigatória, para aplicações de finalidade educacional e não-comercial, de acordo com o modelo de licenciamento Creative Commons adotado pela revista. 